\title{
Aggregation Rate of Charged Colloidal Particles in a Shear Flow: Trajectory Analysis Using Non-linear Poisson-Boltzmann Solution
}

\author{
Takuya SUGIMOTO ${ }^{1} \cdot$ Motoyoshi KOBAYASHI $^{2, *} \cdot$ Yasuhisa ADACHI $^{3}$ \\ ${ }^{1}$ Graduate School of Life and Environmental Sciences, University of Tsukuba \\ (Tennoudai 1-1-1, Tsukuba, Ibaraki 305-8572, Japan) \\ E-mail: s1321138@u.tsukuba.ac.jp \\ ${ }^{2, *}$ Member of JSCE, Dr. (Agr. Sci.), Associate Professor, Faculty of Life and Environmental Sciences, \\ University of Tsukuba (Tennoudai 1-1-1, Tsukuba, Ibaraki 305-8572, Japan) \\ E-mail: kobayashi.moto.fp@u.tsukuba.ac.jp \\ ${ }^{3}$ Dr. (Agr. Sci.), Professor, Faculty of Life and Environmental Sciences, University of Tsukuba \\ (Tennoudai 1-1-1, Tsukuba, Ibaraki 305-8572, Japan) \\ E-mail: adachi.yasuhisa.gu@u.tsukuba.ac.jp
}

\begin{abstract}
We examined aggregation rates of colloidal particles in a shear flow as a function of $\mathrm{KCl}$ (potassium chloride) concentration at different shear rates. The analysis was based on the trajectory analysis with non-linear Poisson-Boltzmann (PB) solution that calculates the double layer force between highly charged particles. The trajectory analysis here is performed without any adjustable parameters. The PB solution enables us to analyze the experimental data of orthokinetic aggregation of highly charged particles where linearized PB solution is not valid. It should be noted that the comparison of the calculation with the experimental data of aggregation rates in a simple shear flow in the presence of the double layer repulsion had never been attempted until we analyzed. The theoretical calculation with trajectory analysis qualitatively describes the experimental data. However, theoretical values of critical coagulation concentration (CCC) being a bending point of capture efficiencies plotted against the $\mathrm{KCl}$ concentration, and capture efficiencies in the presence of double layer force are not quantitatively consistent with experimental ones. These discrepancies might be caused by the additional forces and charge heterogeneity which are not included in the present calculation.
\end{abstract}

Key Words: coagulation, capture efficiency, trajectory analysis, non-linear Poisson-Boltzmann solution

\section{INTRODUCTION}

A significant amount of colloidal particles such as clay minerals and natural organic matters can be found in the natural water and turbid water generated by a heavy rain. The colloidal particles have some important characters; small in size, a large amount of reactive surface sites, and charged surfaces. These natures provide colloidal particles with the ability to adsorb contaminants such as heavy metal and agricultural chemicals. Once these particles are transported in the groundwater, they play an important role to control transport phenomena in water environment ${ }^{1)}$. Colloidal particles aggregate in accordance with the change of chemical and hydrodynamic conditions around the particles. The aggregation of colloidal particles increases the size of transport unit. As a consequence, the transport properties also largely change ${ }^{2)}$. Such changes usually occur in a flow field in water environment. Therefore, understanding the aggregation process of the colloidal particles in the flow field is important to control the transport phenomena of colloidal particles in solid-liquid separation processes and in the prediction of spreading of contaminants in water environment.

Aggregation process is basically determined by the physicochemical interaction and the collision mode between the particles ${ }^{3)}$. The former is mainly composed of the van der Waals (vdW) attraction and the electrical double layer repulsion. The net force can be described as the sum of these interaction forces according to the classical theory by the Derjaguin-Landau-Verwey-Overbeek (DLVO), so-called the DLVO theory ${ }^{4), 5)}$. The collision mode is divided into the collision due to Brownian motion in quiescent fluid and the velocity difference in fluid such as laminar shear and turbulent flow ${ }^{6,7), 8,99,10), 11)}$.

Theoretical formulation for aggregation kinetics in Brownian motion and a laminar shear flow was undertaken by Smoluchowski ${ }^{12)}$. Unfortunately, Smolu- 
chowski's formulation neglects any interactions although the colliding particles hydrodynamically and physicochemically interact with each other in more realistic system. Without any interaction, the trajectory of colliding particles is rectilinear. On the contrary, the collision trajectory with inter-particle interaction becomes curvilinear. Taking into account the physicochemical and hydrodynamic interactions, one can modify the Smoluchowski collision rate $Z_{\text {smol }}$ for the same particles with a radius $R$ in a simple shear flow with a shear rate of $G$ as

$$
Z=\alpha Z_{\text {smol }}
$$

where $Z_{\text {smol }}=32 R^{3} G N_{0} / 3, N_{0}$ and $\alpha$ are the initial total number concentration of the particles and a capture efficiency, respectively. The capture efficiency $\alpha$ reflects the effect of hydrodynamic and physicochemical interactions between the particles on the aggregation rate. The capture efficiency had been numerically calculated with trajectory analysis by many researchers ${ }^{13), 14), 15), 16), 18), 19), 20), 21), 22) \text {. }}$

So far, however, the previous researchers had computed the trajectory by using the double layer repulsion based on the linearized Poisson-Boltzmann equation, which is only applicable under the condition where electric potential between the particle surfaces is low. That is, the previously calculated results can not be applied to the aggregation of particles with high surface potential often faced in the realistic case. Moreover, the theoretical calculations with the trajectory analysis in the presence of the double layer repulsion have not been quantitatively compared with the systematic experimental data taken using the well-characterized colloidal particles, although the kinetic theory in Brownian coagulation has shown that it is quantitatively consistent with the experimental data in some limited conditions ${ }^{23)}$. Here, we focus on the experimental data by Sato $e t a l .{ }^{24)}$. They measured the capture efficiencies as a function of $\mathrm{KCl}$ concentration in a simple shear flow using the well-characterized and highly charged latex particles. Although their experimental data are systematically taken in well-defined system and include the rates in the presence of the repulsive double layer forces, their data have not been substantially analyzed due to the problem of the previous calculation which assumes the low potential condition in the evaluation of the electrical repulsion. Therefore, the kinetics of aggregation in flow fields has not yet been subjected to intense study in spite of its scientific and practical importance.

In the present study, we calculate the capture efficiency of charged colloidal particles in a simple shear flow on the basis of hydrodynamic trajectory analysis incorporating the double layer repulsion with the exact non-linear Poisson-Boltzmann solution in the symmetrical electrolyte solution. Furthermore, from the comparison between theoretical and experimental values in the well-defined system, we discuss the validity of the theory with trajectory analysis for the shear-induced aggregation. It should be noted that such comparisons have never been attempted in the presence of the double layer repulsion.

\section{THEORY}

\section{(1) Electrophoretic mobility and zeta potential}

Electrophoresis has been extensively used to study the charging behavior of colloidal particles ${ }^{23), 25)}$. We thus adopted the electrophoresis to evaluate the charging properties of colloidal particles, such as surface charge density and potential, through the analysis of experimental data by a standard electrokinetic model as described below.

With the Poisson-Boltzmann equation in 1:1 (symmetrical) electrolyte solution, the surface charge density $\sigma$ can be related to the surface potential $\psi_{0, i s o}$ on the isolated particle surface $a^{26)}$

$$
\sigma=\frac{2 \epsilon_{r} \epsilon_{0} \kappa}{\beta e} \sinh \left(\frac{\beta e \psi_{0, i s o}}{2}\right)
$$

with

$$
\kappa=\sqrt{\frac{2 \beta n_{0} e^{2}}{\epsilon_{r} \epsilon_{0}}}
$$

where $\epsilon_{r} \epsilon_{0}, \kappa, n_{0}, \beta=1 /\left(k_{B} T\right)$, and $e$ are the permittivity of the medium, the Debye parameter, the bulk concentration of the electrolyte, the inverse thermal energy, and the elementary charge, respectively. $\kappa^{-1}$ is usually called the Debye length which is the measure of the thickness of electrical double layer.

The electrophoretic mobility $\mu_{m}$ is defined as the ratio of translational velocity of a particle $U$ induced by the applied electric field and the magnitude of the applied electric field $E$ in a solution.

$$
\mu_{m}=\frac{U}{E}
$$

Assuming that the surface charge density is constant which is fulfilled in the present study, the surface potential is determined from Eq.(2). The evaluated surface potential can be used to calculate electrophoretic mobility $\mu_{m}$ if the $\psi_{0, \text { iso }}$ equals to the zeta potential $\zeta$ which is defined as an electrical potential at the outer end of immobile fluid layer near the surface. The electrophoretic mobility $\mu_{m}$ in a solution with a viscosity $\eta$ is often calculated using the HelmholtzSmoluchowski equation, which is also simply called the Smoluchowski equation, given by 


$$
\mu_{m}=\frac{\epsilon_{r} \epsilon_{0}}{\eta} \zeta
$$

This equation is conveniently used to transform the experimental electrophoretic mobility to $\zeta$ because the relationship is linear and simple. However, it is not readily applicable to the case of high $\zeta$ where the relationship between the electrophoretic mobility and $\zeta$ becomes non-linear by the relaxation effect which gives rise to the appearance of the minimum/maximum electrophoretic mobility by decreased translational velocity of a particle due to the deformation of electrical double layer. The deformation is caused by the motion of counter-ion due to the applied electric field in the opposite direction of particle motion and becomes more significant with increasing the surface potential and decreasing the salt concentration. The non-linear relationship has been confirmed using the theoretical computation by O'Brien and White ${ }^{28)}$, and a number of the experimental studies ${ }^{23), 25), 27) . ~}$

Approximate analytical expressions of electrophoretic mobility including the relaxation effect for a sphere have been proposed by Ohshima ${ }^{30), 31)}$. In the present study, we used Ohshima's equations because they are accurate when $\kappa R$ is larger than 10. The large $\kappa R$ means that the particle radius is considerably larger than the thickness of the double layer. This condition is satisfied in the present study. In the 1:1 symmetrical electrolyte solution such as $\mathrm{KCl}$, the analytical expression of electrophoretic mobility $\mu_{m}$ is given by

$$
\begin{aligned}
\mu_{m}=\operatorname{sgn}(\zeta) & \frac{\epsilon_{r} \epsilon_{0}}{\eta}\{|\zeta| \\
& \left.+\left(\frac{1}{e \beta}\right)\left[-\frac{2 F}{1+F} H+\frac{\mathcal{M}}{\kappa R}\right]\right\}
\end{aligned}
$$

with

$$
\begin{gathered}
F=\frac{2}{\kappa R}\left(1+3 m_{+}\right)\{\exp (\beta e|\zeta| / 2)-1\} \\
H=\ln \left[\frac{1+\exp (\beta e|\zeta| / 2)}{2}\right] \\
m_{+}=\frac{2 \epsilon_{r} \epsilon_{0}}{3 \eta e^{2} \beta} \lambda_{+}
\end{gathered}
$$

where $\operatorname{sgn}(\zeta)$ is a function defined as -1 when $\zeta<0$, +1 when $\zeta \geq 0$. Also, $m_{+}$and $\lambda_{+}$are the scaled drag coefficient of counter-ion and the drag coefficient of the counter ion species, respectively. The third term in Eq.(6) including a function of $\mathcal{M}$ can be neglected when $\kappa R$ is considerably large. The detailed functional form of $\mathcal{M}$ is given in the Appendix. The ionic drag coefficient $\lambda_{ \pm}$is defined as

$$
\lambda_{ \pm}=\frac{N_{A} e^{2}\left|z_{ \pm}\right|}{\Lambda_{ \pm}^{0}}
$$

where $N_{A}, z_{ \pm}$, and $\Lambda_{ \pm}^{0}$ are Avogadro's number, the ion valence, the limiting conductance of the each ionic species, respectively. To calculate the electrophoretic mobility, we used the values of the limiting conductance taken from the literature ${ }^{32), 33)}$.

\section{(2) Trajectory analysis}

Capture efficiency is calculated based on trajectory analysis ${ }^{13), 15)}$. Consider two particles with a radius of $R$ and a surface potential of $\psi_{0, \text { iso }}$, which is a electrical potential on the isolated particle surface, in a simple shear flow with a shear rate of $G$. The relative position between the two particles can be described in the Cartesian coordinate $(x, y, z)$ or the spherical coordinate $(r, \theta, \phi)$. The relative velocity of the particles is determined by the following trajectory equations ${ }^{18)}$.

$$
\begin{aligned}
\frac{d r^{*}}{d t^{*}}= & r^{*}\left(1-\mathcal{A}\left(r^{*}, \rho\right)\right) \sin ^{2} \theta \sin \phi \cos \phi \\
& +\frac{\mathcal{C}\left(r^{*}, \rho\right)}{6 \pi \eta G R^{2}}\left(F_{v d W}+F_{e d l}\right) \\
\frac{d \theta}{d t^{*}}= & \left(1-\mathcal{B}\left(r^{*}, \rho\right)\right) \sin \theta \cos \theta \sin \phi \cos \phi \\
\frac{d \phi}{d t^{*}}= & \cos ^{2} \phi-\frac{\mathcal{B}\left(r^{*}, \rho\right)}{2} \cos 2 \phi
\end{aligned}
$$

where $t, r^{*}=r / R, t^{*}=G t$, and $\rho$ are time, dimensionless center-to-center distance of the particles and time, and size ratio, respectively. $\mathcal{A}, \mathcal{B}, \mathcal{C}$ are also hydrodynamic interaction functions that depend on $\rho$ and $r^{*}$.

In the present study, we use the expression of $\mathcal{A}, \mathcal{B}$, $\mathcal{C}$ for $\rho=1$ given by some researchers ${ }^{34), 35), 16), 18), 19),}$ because the particles have the same radius $R$. The near-field functions of $\mathcal{A}, \mathcal{B}, \mathcal{C}$ are described as ${ }^{18)}$

$$
\begin{aligned}
\mathcal{A}\left(r^{*}, \rho\right) & =1-4.077 h^{*} \\
\mathcal{B}\left(r^{*}, \rho\right) & =0.4060+\frac{0.78}{\ln h^{*}} \\
\mathcal{C}\left(r^{*}, \rho\right) & =4 h^{*}\left(1+1.34 h^{*} \ln h^{*}\right)
\end{aligned}
$$

where $h^{*}=r^{*}-\rho-1$ is the dimensionless surface separation distance. Also, the far-field functions of those are shown as ${ }^{34), 35)}$

$$
\begin{aligned}
\mathcal{A}\left(r^{*}, \rho\right)= & \frac{5\left(1+\rho^{3}\right)}{2 r^{* 3}} \\
& -\frac{3\left(1+\rho^{5}\right)+5 \rho^{2}(1+\rho)}{2 r^{* 5}} \\
& +\frac{25 \rho^{3}}{r^{* 6}}
\end{aligned}
$$




$$
\begin{aligned}
& \mathcal{B}\left(r^{*}, \rho\right)=\frac{1+\rho^{5}+(5 / 3) \rho^{2}(1+\rho)}{r^{* 5}} \\
& \mathcal{C}\left(r^{*}, \rho\right)=1+\frac{1}{\rho}
\end{aligned}
$$

In the intermediate distance, the interpolated explicit formulas listed by Wang $^{19)}$ are adopted in the present study.

In Eq.(11), $F_{v d W}$ and $F_{e d l}$ are the van der Waals attractive force and the double layer repulsive force, respectively. The van der Waals attraction including the retardation effect is calculated by the following expressions $^{20), 21), 22)}$, that is, if $p<0.590$,

$$
F_{v d W}=-\frac{A_{\mathrm{H}} R}{(1+\lambda) h^{2}} \frac{1+3.54 p}{6(1+1.77 p)^{2}}
$$

and if $p \geq 0.590$,

$$
F_{v d W}=-\frac{A_{\mathrm{H}} R}{(1+\lambda) h^{2}}\left(\frac{2.45}{15 p}-\frac{2.17}{30 p^{2}}+\frac{1.18}{105 p^{3}}\right)
$$

where $h=r-2 R, A_{\mathrm{H}}$, and $p=2 \pi h / \lambda_{\mathrm{L}}$ are surface separation distance between two equal size particles, the Hamaker constant, and dimensionless distance in which $\lambda_{\mathrm{L}}$ is the London wavelength and has been mainly taken $\lambda_{\mathrm{L}}=100 \mathrm{~nm}$. The value of $\lambda_{\mathrm{L}}$ is comparable to the travel distance of light during one rotation of a Bohr atom electron; $c / \nu \approx 100 \mathrm{~nm}$ where $c$ and $\nu$ are the speed of light and the frequency of rotation of a Bohr atom electron ${ }^{17)}$.

In symmetrical ( $z: z$ type) electrolyte solutions such as $\mathrm{KCl}, F_{\text {edl }}$ can be calculated by using the Derjaguin approximation as follows ${ }^{36)}$ :

$$
F_{e d l}=\frac{2 \pi n_{0} R}{\beta} \int_{h}^{\infty}\left(\cosh \left(\beta e \psi_{m}(L)\right)-1\right) d L
$$

where $n_{0}, \beta=1 /\left(k_{B} T\right), e$, and $\psi_{m}(L)$ are the bulk concentration of the electrolyte, the inverse thermal energy, the elementary charge, and the mid-plane potential between two similar plates with a plate-toplate distance of $L$, respectively. If the electrolyte is symmetrical, $\psi_{m}(L)$ can be related to the surface charge density $\sigma$ by $^{36)}$

$$
\sigma=\frac{\epsilon_{r} \epsilon_{0} \kappa}{\beta e} \frac{\exp \left(2 \beta e \psi_{m}\right)-1}{\exp \left(\beta e \psi_{m} / 2\right)} \frac{\operatorname{sn}(\nu \mid m)}{\operatorname{cn}(\nu \mid m) \operatorname{dn}(\nu \mid m)}
$$

where $\epsilon_{r} \epsilon_{0}$ and $\kappa=\sqrt{2 \beta n_{0} e^{2} /\left(\epsilon_{r} \epsilon_{0}\right)}$ are the permittivity of the medium, and the Debye parameter, respectively. $\operatorname{sn}(\nu \mid m), \operatorname{cn}(\nu \mid m), \operatorname{cd}(\nu \mid m)$ are the Jacobian elliptic functions of argument $\nu=\kappa L /\left[4 \exp \left(\beta e \psi_{m} / 2\right)\right]$ and parameter $m=$ $\exp \left(2 e \beta \psi_{m}\right)$.

The temporal change of relative position between two colliding particles, which means the colliding trajectory, can be calculated based on Eqs.(11)-(13) by the forth Runge-Kutta method. Changing released points of a particle from $x-z$ plane at $y^{*}=y / R=$ -100 in the velocity field $u_{y}=G x$, each trajectory from a release point is judged whether aggregation occurs or not in a deterministic way. The calculation was stopped when the relative position of the particles resulted in one of the following three cases as (i) $r^{*}-\lambda-1<\epsilon^{*}=\epsilon / R$, where $\epsilon$ is the minimum separation, (ii) $\phi>\pi / 2$, or (iii) $y^{*}=10$. The former two cases (i) and (ii) correspond to aggregation. The last case (iii) means that each particle is separated and no aggregation occurs. In the present study, $\epsilon^{*}=10^{-7}$ was adopted to avoid computational divergence $^{20)}$. The boundary between aggregation or not on $x-z$ plane at the released point, $x_{c}(z)$ and the maximum of $z$ on the boundary, $z_{\max }$ can be evaluated with the trajectory analysis described above. With the $x_{c}(z)$ and $z_{\max }$, the particle collision rate $Z$ in Eq.(1) is calculated by integrating the particle flux passing through inner region of the boundary given by

$$
\begin{aligned}
Z & =4 N_{0} G \int_{0}^{z_{\max }} \int_{0}^{x_{c}\left(z^{\prime}\right)} x^{\prime} d x^{\prime} d z^{\prime} \\
& =2 N_{0} G \int_{0}^{z_{\max }}\left[x_{c}\left(z^{\prime}\right)\right]^{2} d z^{\prime}
\end{aligned}
$$

From Eq.(1), Eq.(24) and the Smoluchowski collision rate $Z_{\text {smol }}$, the capture efficiency $\alpha$ is calculated as follows

$$
\alpha=\frac{3}{16} \int_{0}^{z_{\max }^{*}}\left[x_{c}^{*}\left(z^{*}\right)\right]^{2} d z^{*}
$$

where $z^{*}=z / R, z_{\text {max }}^{*}$ and $x_{c}^{*}\left(z^{*}\right)=x_{c} / R$.

\section{EXPERIMENTS}

\section{(1) Materials}

In the present study, we analyze the experimental data of aggregation rate of polystyrene sulfate latex (PSL) particles with two different diameters of 1.96 and $2.8 \mu \mathrm{m}$ by Sato et $a{ }^{24)}$. The smaller particles were synthesized by means of surfactant-free aqueous polymerization. The larger particles were purchased from Interfacial Dynamic Corporation. These PSL particles with the density of $1.055 \mathrm{~g} / \mathrm{cm}^{3}$ are spherical, monodisperse and have the sulfate headgroups on the surface which are strong acid. The surfaces are thus considered to be negatively charged with a constant surface charge density irrespective of $\mathrm{pH}$ and ionic strength. On one hand, the magnitude of surface potential decreases with increasing ionic strength by screening effect. The surface charge densities for the larger and smaller particles were known to be $\sigma=-70 \mathrm{mC} / \mathrm{m}^{2}$ reported by the manufacturer and $\sigma=-60 \mathrm{mC} / \mathrm{m}^{2}$ taken from the literature ${ }^{24)}$, re- 
spectively. The ionic strength was controlled by $\mathrm{KCl}$ solution. More details of these particles are found in elsewhere $^{24)}$. All experiments were carried out at 20 ${ }^{\circ} \mathrm{C}$.

\section{(2) Electrophoresis}

Electrophoretic mobility of these particles was measured as a function of $\mathrm{KCl}$ concentration by using a laser Doppler velocimetry setup (ZetaSizer Nano ZS and 2000, Malvern) to confirm the validity of the standard electrokinetic model described above. The experimental procedures are as follows. Firstly, the colloidal suspensions were prepared by mixing the required amount of stock latex suspension, $\mathrm{KCl}$ solution, and pure water. Secondary, the suspensions were injected into the cell for electrophoretic measurement. Finally, the cell was set in the instrument and the electrophoretic mobility was measured.

\section{(3) Aggregation in a shear flow}

Aggregation experiments in a simple shear flow were performed by using a Couette flow device composed of two concentric cylinders. The Couette flow device generates steady laminar shear flows in the gap between the two cylinders by rotating the outside cylinder. Changing the revolution speed of the outer cylinder $\Omega$, the averaged shear rate in the gap $G_{\text {ave }}$ can be controlled and calculated by

$$
G_{\text {ave }}=\frac{4 \Omega}{\left(\frac{1}{r_{1}^{2}}-\frac{1}{r_{2}^{2}}\right)\left(r_{2}^{2}-r_{1}^{2}\right)}
$$

where $r_{1}$ and $r_{2}$ are the outer radius of the inside cylinder and the inner radius of the outside cylinder, respectively. The value of $G_{\text {ave }}$ is used as $G$ in Eq.(11) in the trajectory analysis. The experiments were performed at $G=23,46,92 \mathrm{~s}^{-1}$ corresponding Peclet numbers defined by

$$
P e=3 \pi \beta \eta R^{3} G
$$

were larger than 50; the effect of Brownian motion was negligible compared to fluid motion.

The aggregation experiments of the particles were carried out as a function of $\mathrm{KCl}$ concentration. The required amounts of latex suspension, $\mathrm{KCl}$ solution, and pure water were mixed and immediately poured into the gap in the Couette flow device. Then, the suspension was subjected to a shear flow with three shear rates of $G=23,46,92 \mathrm{~s}^{-1}$. The rotation was stopped in certain period of time and the suspension was sampled to measure the total particle number concentration $N(t)$ at elapsed time $t$ by a Coulter particle counter. From the measured relationship between $N(t)$ and $t$, the capture efficiency $\alpha$ can be obtained with the following expression by assuming

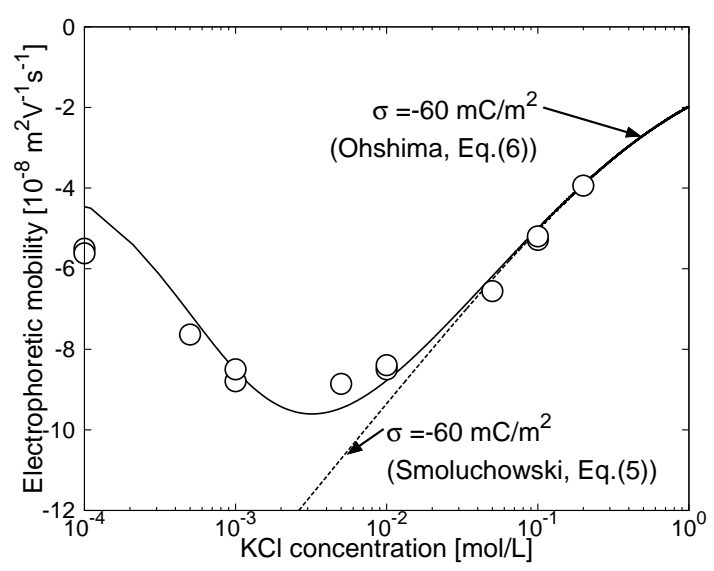

Fig.1 The relationship between electrophoretic mobility and $\mathrm{KCl}$ concentration for the particles with the diameter of $2 R=1.96 \mu \mathrm{m}$ : Open circles and lines are experimental values and theoretical values calculated by standard electrokinetic model, respectively.

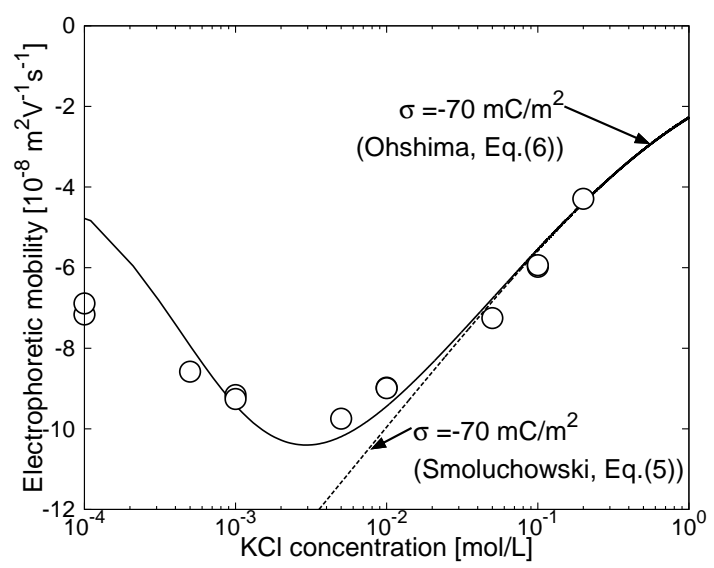

Fig.2 The relationship between electrophoretic mobility and $\mathrm{KCl}$ concentration for the particles with the diameter of $2 R=2.8 \mu \mathrm{m}$ : Open circles and lines are experimental values and theoretical values calculated by standard electrokinetic model, respectively.

the constant volume fraction of particles in the early stage of aggregation process ${ }^{24}$

$$
\ln \left[\frac{N(t)}{N_{0}}\right]=-\alpha \frac{4 \phi G}{\pi} t
$$

where $\phi$ is the volume fraction of particles in the suspension. These experiments were performed in the low volume fraction $\phi$ of $0.4-3.8 \times 10^{-5}$. Therefore, we assume that the effect of volume fraction on the flow field is negligible. 


\section{RESULTS AND DISCUSSION}

\section{(1) The electrophoretic mobility}

In Figs. 1 and 2, measured electrophoretic mobilities (open circles) are shown with theoretical ones (lines). The solid and broken lines are the theoretical calculation with the relaxation effect (Eq.(6) proposed by Ohshima) and without the relaxation (Eq.(5) proposed by Smoluchowski), respectively. Surface charge densities $\sigma$ of $-60 \mathrm{mC} / \mathrm{m}^{2}$ for the smaller latex and $-70 \mathrm{mC} / \mathrm{m}^{2}$ for the larger particles were taken from the literature ${ }^{24)}$. From Figs. 1 and 2, we see that the experimental values of electrophoretic mobility decrease with decreasing the $\mathrm{KCl}$ concentration and show a minimum value, indicating that the ion relaxation effect becomes significant with increasing the surface potential and with the decrease of the $\mathrm{KCl}$ concentration. The theoretical values including the ion relaxation effect agree with the experimental ones whereas the calculations without the relaxation effect largely deviate from the experimental values at low $\mathrm{KCl}$ concentration. In the lowest $\mathrm{KCl}$ concentration, where the surface potential is the highest, the Ohshima theory slightly overestimates the experimental ones. The discrepancies are not significant compared to the Smoluchowski's theory. But there is the difference between Ohshima theory and experiment. Similar differences have been observed by some other researchers at low ionic strength ${ }^{11), 23)}$. In the case of low ionic strength and high surface potential, the ion relaxation effects are significant. As a result, the electrophoretic mobility shows the minimumu value. While the theory including the relaxation captures the mobility minimum, differences exist between the experimental data and the theoretical values in such conditions. The discrepancy is probably caused by the incompleteness of the ion relaxation theory. At high surface potential and low salt concentration, the surface strongly attracts and concentrates the counter-ions near the surface. Because the theory does not include the effect of finite size of ions, the exact description of the layer structure and mobility of accumulated ion near the surface might be still lacking. In any case, from the analysis, we conclude that the standard electrokinetic model and the charge densitiy are valid for both particles, in particular, in the $\mathrm{KCl}$ concentration larger than $10^{-3} \mathrm{M}$. Therefore, the surface charge densities used in the electrophoretic mobility are used for the trajectory analysis in the following section.

\section{(2) Capture efficiency in a shear flow}

Measured and theoretical capture efficiencies for different shear rates are shown in Fig. 3 for smaller particles $(2 R=1.96 \mu \mathrm{m})$ and Fig. 4 for larger particles $(2 R=2.8 \mu \mathrm{m})$. Open circles and solid lines

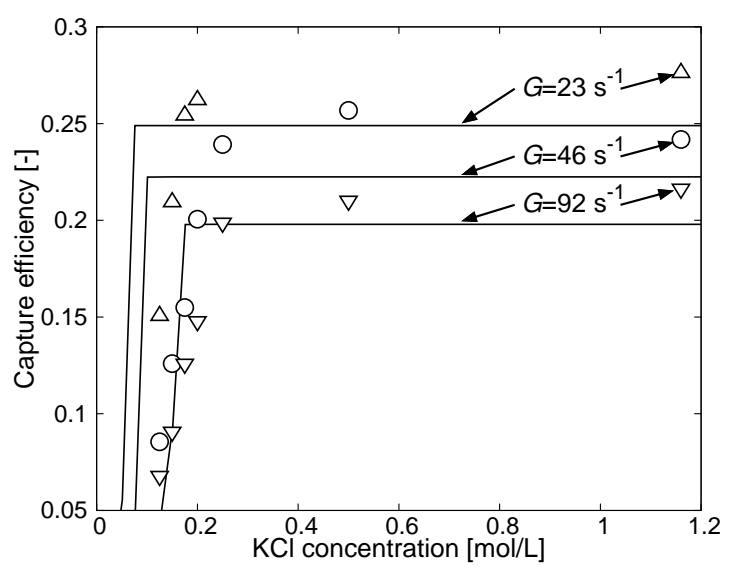

Fig.3 Capture efficiency vs. $\mathrm{KCl}$ concentration for the particles with the diameter of $2 R=1.96 \mu \mathrm{m}$ : Open circles and solid lines are experimental values taken from Sato et al. $^{24)}$ and theoretical values calculated by trajectory analysis with the exact PB solution, respectively.

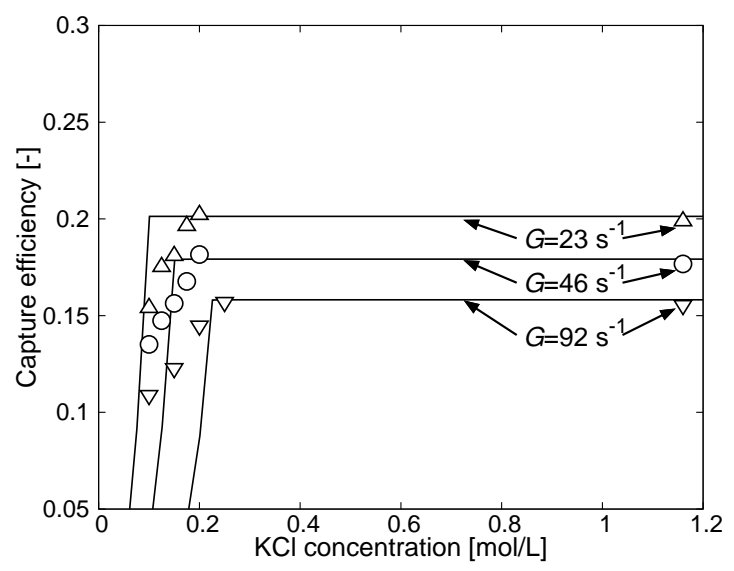

Fig.4 Capture efficiency vs. $\mathrm{KCl}$ concentration for the particles with the diameter of $2 R=2.8 \mu \mathrm{m}$ : Open circles and solid lines are experimental values taken from Sato et ll $^{24)}$ and theoretical values calculated by trajectory analysis with the exact PB solution, respectively.

are experimental and theoretical values, respectively. The theoretical values obtained by using the trajectory analysis with non-linear Poisson-Boltzmann equation are calculated with the Hamaker constant of $A_{\mathrm{H}}=$ $2.0 \times 10^{-21} \mathrm{~J}$ for both particles. This value of $A_{\mathrm{H}}$ for polystyrene latex particles has been confirmed to agree with the Lifshitz theory considering the effects of surface roughness and have been verified by the direct force measurement by atomic force microscope ${ }^{37)}$. When we calculated the repulsive double layer force in this study, the surface charge density of the particles was assumed to be constant as the electrostatic boundary condition. This boundary condition is valid for the used particle surfaces bearing the strong acid groups, whose deprotonation is independent of $\mathrm{pH}$ and the surface separation, which means that protonation occurs to remain the surface 
equilibria when the surfaces approach to contact. It should be emphasized that the double layer repulsion was calculated without adjustable parameters, since the validity of the surface charge densities was confirmed by describing the experimental electrophoretic mobility with the standard electrokinetic model.

From Figs. 3 and 4, we find that the calculated and measured capture efficiencies decrease with decreasing the $\mathrm{KCl}$ concentration, demonstrating that the aggregation is impeded by the increased double layer repulsion. Furthermore, the calculated capture efficiencies qualitatively describe the experimental trend. In particular, from both figures, we find that, in both experiment and theoretical calculation, (1) CCC shifts to higher $\mathrm{KCl}$ concentration and (2) the dependencies of capture efficiencies on the $\mathrm{KCl}$ concentration slightly become more gradual as the shear rate increases. In addition, more gradual slope in the relationship between capture efficiency and $\mathrm{KCl}$ concentration below CCC is observed for the larger particle. This trend is also reproduced by the trajectory analysis. The agreement between theory and experiment in the trends of capture efficiency found in this study provides an evidence of the usefulness of the trajectory analysis for the study of shear-induced aggregation. However, the calculated values of CCC do not perfectly agree with experimental ones. Also, in the slow aggregation regime below $\mathrm{CCC}$, the capture efficiencies are not in quantitative agreement with the experimental ones. Moreover, theoretical capture efficiencies in the absence of double layer force in Fig. 3 are slightly lower than the experimental values. These discrepancies might be caused by the additional nonDLVO forces such as hydration force and charge heterogeneity ${ }^{23), 17)}$. Such non-DLVO forces should be taken into consideration in the trajectory analysis in future studies.

\section{CONCLUSION}

We first analyzed capture efficiency of a negatively charged sulfate latex particles as a function of $\mathrm{KCl}$ concentration and shear rate on the basis of the trajectory analysis. In the calculation of the double layer repulsion with the non-linear Poisson-Boltzmann equation, the constant charge (CC) boundary condition was used, since it is valid for the latex particles bearing strong acid (sulfate) groups whose charges are independent of $\mathrm{pH}$. The validity of surface charge densities as an input value to calculate the repulsive force was confirmed by the electrophoretic measurements with the analysis by the standard electrokinetic model. Calculated capture efficiencies were in reasonable agreement with experimental values taken from the previous study ${ }^{24)}$. Our finding suggests that the trajectory analysis is valuable for the study on the aggregation in flow fields. However, the quantitative differences between measured and calculated capture efficiencies were observed in the presence of the double layer repulsion even with the use of the exact Poisson-Boltzmann solution. The difference might be caused by additional non-DLVO forces and charge heterogeneity ${ }^{23), 17)}$ which are not included in the present analysis.

ACKNOWLEDGMENT: We are grateful for the financial support from JSPS KAKENHI (22248025, 23688027)

\section{APPENDIX A Description of $\mathcal{M}$}

In the present study, the theoretical values of electrophoretic mobility are calculated by using the approximate analytic expression suggested by Ohshima ${ }^{30)}$. Function $\mathcal{M}$ in Eq.(6) is given by

$$
\begin{aligned}
\mathcal{M}= & -18\left(t+\frac{t^{3}}{9}\right) K \\
& +\frac{15 F}{1+F}\left(t+\frac{7 t^{2}}{20}+\frac{t^{3}}{9}\right) \\
& -6\left(1+3 m_{-}\right)(1-\exp (-|\tilde{\zeta}| / 2)) I \\
& +\frac{12 F}{(1+F)^{2}} H+\frac{9|\tilde{\zeta}|}{1+F}\left(m_{-} I+m_{+} H\right) \\
& -\frac{36 F}{1+F}\left(m_{-} I^{2}+\frac{m_{+}}{1+F} H^{2}\right)
\end{aligned}
$$

with

$$
\begin{aligned}
\tilde{\zeta} & =\beta e \zeta \\
t & =\tanh (|\tilde{\zeta}| / 4) \\
K & =1-\frac{25}{3(\kappa R+10)} \exp \left(-\frac{\kappa R}{6(\kappa R-6)}|\tilde{\zeta}|\right)
\end{aligned}
$$

$$
I=\ln \left[\frac{1+\exp (-|\tilde{\zeta}| / 2)}{2}\right]
$$

where $\tilde{\zeta}$ is the non-dimensional $\zeta$ potential. $F$ and $H$ have been already defined by Eq.(7) and Eq.(8), and $m_{-}$is the scaled drag coefficient of co-ion defined by Eq.(9) replacing $\lambda_{+}$to $\lambda_{-}$.

\section{REFERENCES}

1) Elimelech, M., Jia, X., Gregory, J., and Williams, R.: Particle deposition and aggregation: measurement, modelling and simulation, Butterworth-Heinemann, 1998.

2) McAnally, W. H., and Mehta, A. J.: Aggregation rate of fine sediment, Journal of Hydraulic Engineering, Vol.126, pp.883-892, 2000.

3) Russel, W. B., Saville, D. A., and Schowalter, W. R.: Colloidal dispersions, Cambridge University Press, 1992. 
4) Derjaguin, B., and Landau, L.: Acta Physicochem, U.S.S.R., Vol.14, pp.633-662, 1941.

5) Verwey, E. E. J. W., Overbeek, J. T. G., and Overbeek, J. T. J. T. G.: Theory of the stability of lyophobic colloids, DoverPublications. com, 1999.

6) Adachi, Y.: Dynamic aspects of coagulation and flocculation, Adv. Colloid and Interface Sci., Vol.56, pp.1 -31, 1995.

7) Kobayashi, M., Maekita, T., Adachi, Y., and Sasaki, H.: Colloid stability and coagulation rate of polystyrene latex particles in a turbulent flow, International Journal of Mineral and Processing, Vol.73, pp.177-181, 2004.

8) Brunk, B. K., Koch, D. L., and Lion, L. W.: Observations of coagulation in isotropic turbulence, J. Fluid Mech., Vol.371, pp.81-107, 1998.

9) Brunk, B. K., Koch, D. L., and Lion, L. W.: Hydrodynamic pair diffusion in isotropic random velocity fields with application to turbulent coagulation, Physics of Fluids, Vol.9, pp.2670-2691, 1997.

10) Winterwerp, J. C.: A simple model for turbulence induced flocculation of cohesice sediment, Journal of Hydraulic Research, Vol.36, pp.309-326, 1998.

11) Sugimoto, T., Kobayashi, M., Adachi, Y.: The effect of double layer repulsion on the rate of turbulent and Brownian aggregation: experimental consideration, Colloids and Surfaces A, Vol.443, pp.418-424, 2014.

12) Smoluchowski, M. V.: Versuch einer mathematischen Theorie der Koagulationskinetik kolloider Lsungen, Z. Phys. Chem., Vol.92, pp.129-168, 1917.

13) Zeichner, G. R., and Schowalter, W. R.: Use of trajectory analysis to study stability of colloidal dispersions in flow fields, AlChE J., Vol.23, pp.243-254, 1977.

14) Schowalter, W. R.: Stability and coagulation of colloids in shear fields, Annual Review of Fluid Mechanics, Vol.16, pp.245-261, 1984.

15) Van de Ven, T. G. M., and Mason, S. G.: The microrheology of colloidal dispersions VII. Orthokinetic doublet formation of spheres, Colloid Polymer Sci., Vol.255, pp.468-479, 1977.

16) Arp, P. A., and Mason, S. G.: The kinetics of flowing dispersions VIII. doublets of rigid spheres (theoretical), J. Colloid Interface Sci., Vol.61, pp.21-43, 1977.

17) Israelachvili, J. N.: Intermolecular and Surface Forces Third Edition, Academic press, 2011.

18) Adler, P. M.: Interaction of unequal spheres I. hydrodynamic interaction: colloidal forces, J. Colloid Interface Sci., Vol.84, pp.461—473, 1981.

19) Wang, Q.: A study on shear coagulation and heterocoagulation, J. Colloid Interface Sci., Vol.150, pp.418 -427, 1992.

20) Vanni, M., and Baldi, J. B.: Coagulation efficiency of colloidal particles in shear flow, Avd. Colloid Interface Sci., Vol.97, pp.151-177, 2002.

21) Kobayashi, M.: Aggregation of unequal-sized and oppositely charged colloidal particles in a shear flow, J. Applied Mechanics, JSCE, Vol.11, pp.517-523 2008.
22) Kobayashi, M.: Kinetics of shear coagulation of oppositely charged particles: A trajectory analysis, Theoretical and applied mechanics Japan, Vol.56, pp.267 -272, 2008.

23) Behrens, S. H., Christl, D. I., Emmerzael, R., Schurtenberger, P., and Borkovec, M.: Charging and aggregation properties of carboxyl latex particles: experiments versus DLVO theory, Langmuir, Vol.16, pp.2566-2575, 2000.

24) Sato, D., Kobayashi, M., and Adachi, Y.: Capture efficiency and coagulation rate of polystyrene latex particles in a laminar shear flow: Effects of ionic strength and shear rate, Colloids and Surfaces A, Vol.266, pp.150-154, 2005.

25) Ottewil, R. H., and Shaw, J. N.: Electrophoretic studies on polystyrene latices, J. Electroanalytical Chemistry and Interfacial Electrochemistry, Vol.37, pp.133 -142, 1972.

26) Ohshima, H.: Biophysical chemistry of biointerfaces, John Wiley and Sons, 2011.

27) Kobayashi, M.: Electrophoretic mobility of latex spheres in the presence of divalent ions: experiments and modeling, Colloid Polymer Sci., Vol.286, pp.935 -940, 2008.

28) O'Brien, R. W., and White, L. R.: Electrophoretic mobility of a spherical particle, J. Chem Soc Faraday Trans 2, Vol.74, pp.1607-1626, 1978.

29) O'Brien, R. W., and Hunter, R. J.: The electrophoretic mobility of large colloidal particles, Can. J. Chem., Vol.59, pp.1878-1887, 1981.

30) Ohshima, H., Healy, T. W., and White, L. R.: Approximate analytic expressions for the electrophoretic mobility of spherical colloidal particles and the conductivity of their dilute suspensions, J. Chem Soc Faraday Trans 2, Vol.79, pp.1613-1628, 1983.

31) Ohshima, H.: Approximate expression for the electrophoretic mobility of a spherical colloidal particle in a solution of general electrolytes, Colloids and Surfaces A, Vol.267, pp.50-55, 2005.

32) Lide, D. R., (ed.): CRC handbook of chemistry and physics, CRC press, Boca Raton, 2004.

33) The Chemical Society of Japan (ed.): Kagaku Binran, Maruzen, Tokyo, 2004.

34) Batchelor, G. K., and Green, J. T.: The hydrodynamic interaction of two small freely-moving spheres in a linear flow field, J. Fluid Mech., Vol.56, pp.375400, 1972.

35) Spielman, L. A.: Viscous interactions in Brownian coagulation, J. Colloid Interface Sci., Vol.33, pp.562 $-571,1970$.

36) Behrens, S. H., and Borkovec, M.: Electrostatic interaction of colloidal surfaces with variable charge, $J$. Phys. Chem. B, Vol.103, pp.2918-2928, 1999.

37) Elzbieciak-Wodka, M., Popescu, M. N., RuizCabello, F. J. M., Trefalt, G., Maroni, P., and Borkovec, M.: Measurements of dispersion forces between colloidal latex particles with the atomic force microscope and comparison with Lifshitz theory, $J$. Chem. Phys., Vol.140, 104906, 2014. 